\title{
What Do Psychology and Civil Engineering have in Common to Promote Well-Being and Excellence?
}

\section{Said Easa}

Ryerson University

Maureen Reed

Ryerson University

Frank A. Russo

Ryerson University

Please Cite:

Easa, S., Reed, M., \& Russo, F. A. (2008). What do psychology and civil engineering have in common to promote well-being and excellence? Canadian Civil Engineer, 25.4, 14-18.

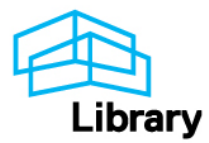



and safety of civil engineering systems as well as aiding education and training.

The purpose of this article is to discuss the role that psychology plays in civil engineering and to highlight some emerging and interesting applications. Before addressing these aspects, a brief description of some basic fundamentals of psychology is presented.

\section{PSYCHOLOGY FUNDAMENTALS}

Psychology is a broad and diverse field involving over 60 subfields, and the number is constantly growing and evolving. ${ }^{2}$ There is currently no universal classification of the psychology areas. However, for purposes of this article, psychology is divided into two broad areas: clinical and experimental. Clinical psychologists assess, diagnose, treat, and prevent mental, emotional, and behavioural disorders, and conduct research for the purpose of understanding dysfunction and promoting well-being. Experimental psychologists approach psychology as one of the natural sciences, and therefore are concerned with basic and applied research in all aspects of human behaviour.

There are several categories of experimental psychology that would be of interest to civil engineers. These are: (1) cognitive psychology (human thought processes, including awareness, perception, reasoning, judgment, and decision making), (2) biopsychology (the relationship between the body systems and behavior), (3) industrial/organizational psychology (workplace behavior to increase productivity, efficiency, and safety), (4) developmental psychology (psychological changes that occur across the life-span), (5) educational psychology (the processes of learning, remembering, and thinking in order to design, develop, and evaluate materials and procedures for education and training), (6) environmental psychology (how cognitive and psychological processes affect the design of environments), (7) social psychology (how a person's thoughts, behaviors, and feelings are affected by the presence of other people), and (8) health psychology (how biological, psychological, and social factors affect health and illness). Most experimental psychologists cross a number of these categories.

In general, experimental psychologists (especially in cognition) are interested in how individuals perceive and interpret the environment. Their training is scientific in

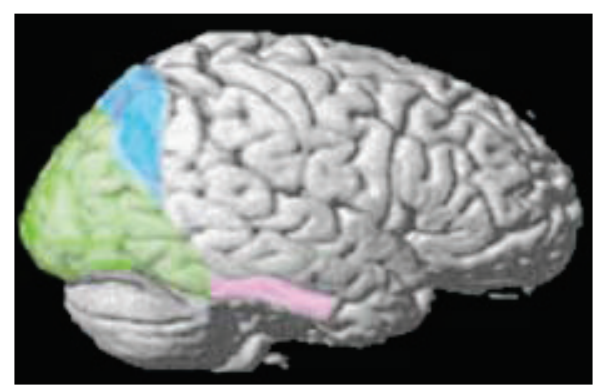

A) BRAIN

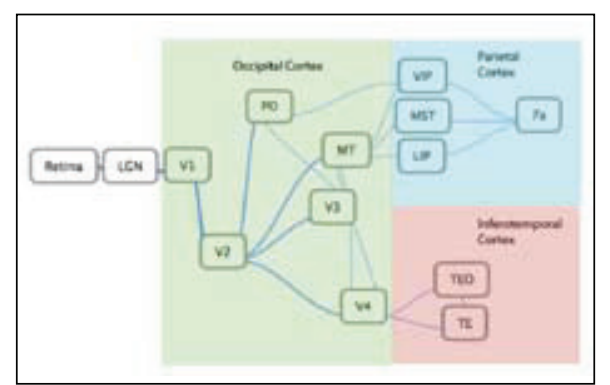

B) MIND (NEURAL NETWORK)

FIGURE 1. Information processing in psychology involves the brain (hardware) and the mind (software). Panel A shows a side view of the brain. The occipital cortex (green) lies at the back of the brain and is responsible for primary visual processing. The inferotemporal projections (purple) are primarily responsible for object vision (i.e. "what"), whereas the parietal projections (blue) are primarily responsible for spatial vision (i.e. "where"). A current view of the corresponding neural networks is shown in Panel B.

\section{Psychology}

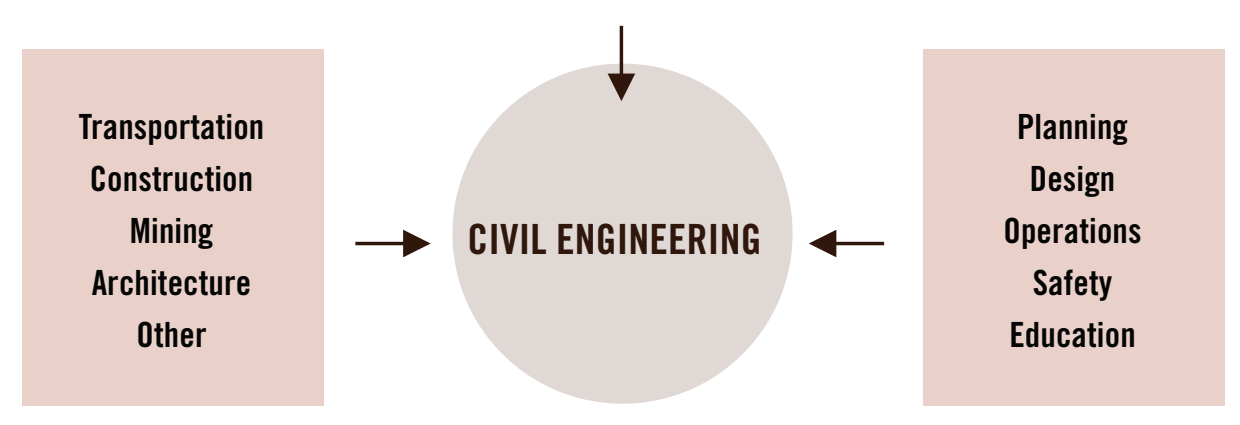

FIGURE 2. Conceptual linkage between psychology and civil engineering.

nature and their expertise is in the design of experiments that are intended to evaluate human performance. It is also not unusual for them to collaborate with engineers. As such, they are frequently involved in experiments to determine how human participants deal with environmental designs. For example, participants are often asked to determine the smallest change that an individual might notice or the limits of the individuals' perceptual abilities.

Engineers and others have elected to work with psychologists to gain insights into human populations. Psychologists often examine the effect of changes in cognitive or perceptual function on everyday abilities for various environments, such as driving, use of equipment, and reading. They may focus on special populations, such as the elderly, accident victims, individuals with disorders, or any group that requires high levels of human performance such, as air traffic controllers. For example, psychologists have worked with the Canadian Space Agency and NASA to understand perceptual processes in microgravity and the effects of extended spaceflight on eye- hand coordination and attention. ${ }^{3}$ Other scientists have examined signal attributes and the interpretation/confusion of signals meant to warn people. Also psychologists develop computer models for artificial intelligence designs that make robotic behaviour more human like. Finally, many psychologists examine human organizations and their effect on memory, decision making, and attention.

Experimental psychology has many tools for addressing issues of human performance. The independent measures collected include reaction time (commonly used where attention is an issue), evoked potentials (brain waves), physiological responses (e.g. heart rate), cognitive tests (e.g. memory, attention, processing speed), and behavioral responses (e.g. eye movement). In particular, information processing in psychology is computational in nature, involving the brain (hardware) and the mind (software) which is represented by a neural network (Fig. 1). This mind-based neural network is the foundation for the well-known neural network estimation techniques commonly used in engineering. 


\section{ERGONOMICS IN INDIVIDUAL CIVIL ENGINEERING AREAS}

Ergonomics has been addressed in several civil engineering areas, including transportation, construction, and mining (Fig. 2). In transportation, ergonomics has been incorporated in the planning, design, operations, and safety of all systems, including highway, public transit, rail transit, bicycles and pedestrians, aviation, and maritime. The Transportation Research Board recently held a workshop to review the developments in human system integration and presented a number of "Best Practice" cases to examine how best to apply behavioural science principles, knowledge, and analytical models. ${ }^{4}$ For example, one case involved operator fatigue and alertness, where programs have been developed to actively manage fatigue in a tailored and non-prescriptive way (i.e. no set maximum hours of service). There was general consensus that success would depend not only on a technically well-designed process, but also on a number of critical elements, such as management commitment, user/stakeholder involvement, qualified personnel, and funding support.

Some interesting work in maritime transportation is worth noting. The U.S. Coast Guard has changed national and international culture to include human factors considerations in all regulatory developments and operating procedures. The need for human factors implementation from a holistic perspective include understanding human perception (e.g. visual limitations), groups (e.g. communication), technology (e.g. human/instrument interactions), organizations (e.g. policies), work practices (e.g. informal rules), society and culture (e.g. socio-political environment), and the physical environment (e.g. vibration). ${ }^{5}$ Future human factors research includes the effect of new enhanced navigation technology and increased automation in the engine room.

In construction, technical regulations and programs have been developed to ensure a basic level of equipment and procedural safety for workers. Human factors engineering programs aim to modify work procedures and equipment to account for the physical and psychological capabilities and limitations of humans. Examples of research topics include the perception of balance at sloped roof surfaces, work compatibility and musculoskeletal disorders, and effect of forklift operation on lower back pain. For information on the extensive array of relevant topics in this area, the reader is referred to various ergonomics journals.

In the mining industry, ergonomics issues include lighting design, physical and postural stress, noise, dust, vibration, and communications. Examples of the implemented strategies are the design of integrated control and monitoring, mining machinery, and rest breaks or rotation to alter the duration

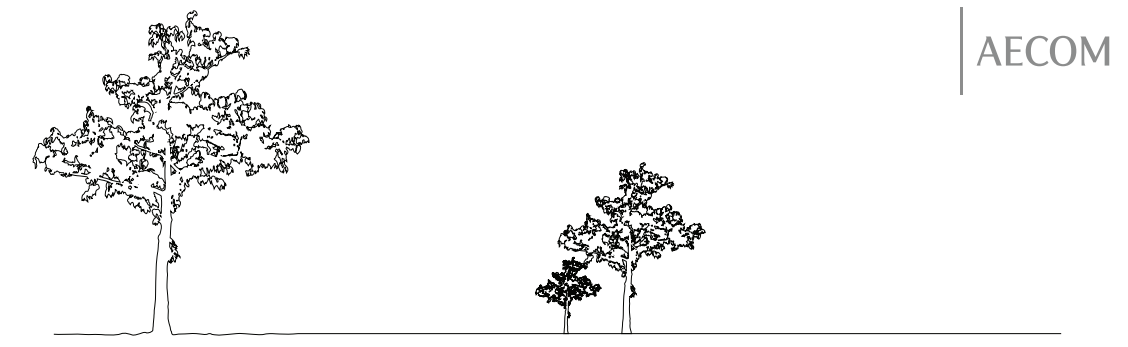

\section{Branching out across Canada.}

AECOM has branched out again, with the recent addition of Earth Tech, Tecsult and TSH to our family of companies. Combined with Gartner Lee, KMK, and UMA, AECOM now has more than 4,000 talented professionals in Canada.

As North America's fastest-growing consulting engineering firm, we have expanded the depth and breadth of our expertise to enhance the full-service solutions we offer to our clients. of exposure to the tasks. Other strategies include publishing an ergonomics information bulletin and establishing awareness of ergonomics among managers. New ergonomically related awards for excellence have been established, such as the Safe-in-Sound award which will be offered in 2009 by the National Institute for Occupational Safety and Health. ${ }^{6}$

In architecture (an area closely related to civil engineering), ergonomics is a key ingredient in the design of buildings and other infrastructure. ${ }^{7}$ Architects focus on the physical features that reflect people's needs/preferences and shape their behavior. For example, in buildings attention is given to the design and arrangement of facilities and associated features, such as lighting, fixtures, and furnishings to develop the most efficient and safer interactions with people. In fact, the relatively new field of environmental psychology is quite related to architecture.

Ergonomics considerations in other civil engineering areas have been limited. In structural engineering, for example, some sporadic research has addressed occupant comfort during wind-induced tall building motion, human-based design approaches to minimize excessive vibrations in residential timber floors, and human error in structural reliability analysis.

It is clear that ergonomics has been considered more prominently in some areas of civil engineering than in others. Nonetheless, managers should place importance on the role of the human in the total system, and knowledge and awareness of ergonomics should become part of the cultural fabric of all civil engineering areas.

\section{EMERGING AND INTERESTING APPLICATIONS}

\section{Aging Driver Mobility}

Addressing aging-driver mobility and safety requires a multidisciplinary approach that should involve the psychology field. The Canadian Council of Motor Transport Administrators has established an "Aging Driver Strategy 2006-2010” to help jurisdictions develop plans and programs to support safe mobility for older Canadians. The strategy addresses key issues, including fitness-based assessment, multi-modal planning, evidence-based policies and programs (e.g. proven best practices and research needs), and partnerships and collaboration. 
To ensure success, the strategy involves not only civil engineers, planners, and driver licensing authorities, but also social support agencies and the medical profession

As for research, it is well known that age causes changes to both perceptual and cognitive mechanisms. Psychologists have recently become interested in the effects of these losses on activities of daily living. Older adults show losses in their ability to process motion, contrast, depth, script, and information, as well as visually search environments. ${ }^{8,9}$ While studies have related cognitive and visual losses to collision frequency, driving self-restrictions, and difficulties in driving maneuvers, the effects of these losses on specific driving situations are largely unknown.

Developmental psychology research has led to two emerging fields in transportation. First, there is a focus on determining the combination of perceptual and cognitive losses that can best predict older driver performance. Such work would help to identify at-risk drivers through perceptual and cognitive tests. Second, the design of driving environments that might compensate for age-related losses has become important. Examples include improved road lighting, in-vehicle collision warning systems, improved sign reflectance, and older driver training. The authors of this article are currently conducting a research project for the Ministry of Transportation Ontario to determine whether improved street lighting can benefit older drivers.

\section{Human Workload}

One emerging area in transportation safety is human workload in the context of the human interface for driver-assist technologies. ${ }^{10}$ While such technologies may be helpful, they may also act as a distracter on the driver. Similar to cell phones, interfaces may divert attention away from the road, particularly when there is a visual component involved that the driver must react to. Emerging technologies are prudently making use of non-visual modalities (e.g. auditory) to minimize visual interference. Nonetheless, for many interfaces, the processing demands are cognitive as much as they are perceptual. Therefore, there exists a need to identify perceptually meaningful signals that do not require extensive cognitive processing.

One promising method that cognitive psychologists have developed to assess

\section{...managers should place importance on the role of the human in the total system, and knowledge and awareness of ergonomics should become part of the cultural fabric of all civil engineering areas.}

the extent of cognitive processing that is required by a secondary task (e.g. warning signal) is to monitor performance across varying levels of attention. If performance in the secondary task is consistent when the demand of the primary task increases, then processing of the secondary task is thought to be not demanding of cognitive resources. The ultimate objective in this research is to develop interfaces and signals that provide meaningful, easily processed information. Other research has addressed the effect of road geometric elements on driver mental workload which represents a promising approach for road design and safety. ${ }^{11}$

\section{Education}

There is currently little attention given to ergonomics in undergraduate civil engineering education. Given the emerging importance of this field in almost all areas of civil engineering, it is recommended that ergonomics material should be explicitly incorporated in all relevant civil engineering courses and should receive more treatment in current core courses on ethics and professionalism.

Furthermore, offering a core cognitive engineering course at the undergraduate level should be explored. A panel at the 2007 Human Factors and Ergonomics Society annual meeting has discussed various issues related to the development of a cognitive engineering textbook. The panel, which consisted of recognized leaders from universities and industry, has discussed related challenges, produced useful ideas about the ideal content of such a book, and prompted continued dialogue on these issues.

\section{Auditory Warnings}

Auditory warnings are useful in a number of civil engineering applications, especially transportation. Psychologists have worked with Transport Canada to examine means of increasing the urgency of auditory warnings without increasing loudness. ${ }^{12}$ Another area in which research may contribute to improved auditory warnings is the design of rumble strips (raised or grooved patterns constructed on specific road elements such as shoulders). This innovation was initially introduced as a measure to combat drift-off-road collisions. When a vehicle makes contact with the rumble strip, a lowtech multisensory alarm is activated that involves auditory (or vibrotactile) stimulation. Ideally, this stimulation will be perceived as urgent enough so as to cause a driver to take appropriate action.

The question of urgency is particularly acute in situations where the driver is drowsy, intoxicated, or under high cognitive load (e.g. talking on cell phone). Further gains in safety may be obtained by optimizing the configuration pattern of rumble strips to increase urgency and reduce reaction time. ${ }^{13}$

Electrophysiology may be combined with behavioral methods to better understand the effectiveness of auditory warnings. For example, galvanic skin (sweat) response provides a useful measure of the physiological reactivity to an auditory warning. Differences in physiological reactivity may provide useful information that helps, for example, in deciding whether a warning was actually perceived.

\section{Macroergonomics}

Macroergonomics, also known as organizational psychology, is among the newest fields in psychology. It focuses on a broad system view of design, examining organizational environments, culture, history, and work goals. ${ }^{2}$ Its objective is to develop an efficient work system that improves productivity and promotes employee satisfaction, health, safety, and commitment. An example is conducting a safety culture review of a rail industry that may involve analyzing the safety management system, reviewing safety training, inspecting records, and interviewing the workforce.

Most universities and organizations have human resources and development units 
with many responsibilities related to macroergonomics. Civil engineers, whether in academia or in practice, ought to take advantage of these units in all planning, design, operations, and safety aspects. Close collaboration with these units can help the system to be more functional and more enjoyable for its people.

\section{CONCLUDING REMARKS}

Collaborations between psychologists and civil engineers benefit both groups. First, psychologists benefit from increased knowledge of environmental design, while civil engineers benefit from the psychologists unique training and knowledge in human information processing. Second, attracting students to graduate studies can be challenging to psychologists and civil engineers. Research collaboration benefits these cross disciplinary fields by offering students a breath of training that would not be received by studying either field in isolation. Finally, while the problems that these groups tackle are research based, they often have applications in discovering solutions for practical problems and directions for future research.
For further information, the reader may consult the website of the International Ergonomics Association (www.iea.cc) which provides information related to the science of ergonomics. The IEA has several technical committees that are of interest to civil engineers, such as Aging, Auditory Ergonomics, Building and Construction, Ergonomics in Design, Organizational Design and Management, Psychophysiology in Ergonomics, and Transport.

We hope that this article will raise awareness about the critical importance of ergonomics in civil engineering and encourage collaboration between psychologists and civil engineers to promote well-being and excellence.

\section{REFERENCES}

1 Chebykin, O.Y., Bedny, G.Z., and Karwowski, W. (eds.) (2008). Ergonomics and psychology: Developments in theory and practice. CRC Press, Boca Raton, Florida.

2 Wikipedia (2008). Psychology. Internet: en.wikipedia.org/wiki/Psychology (accessed August 5, 2008).

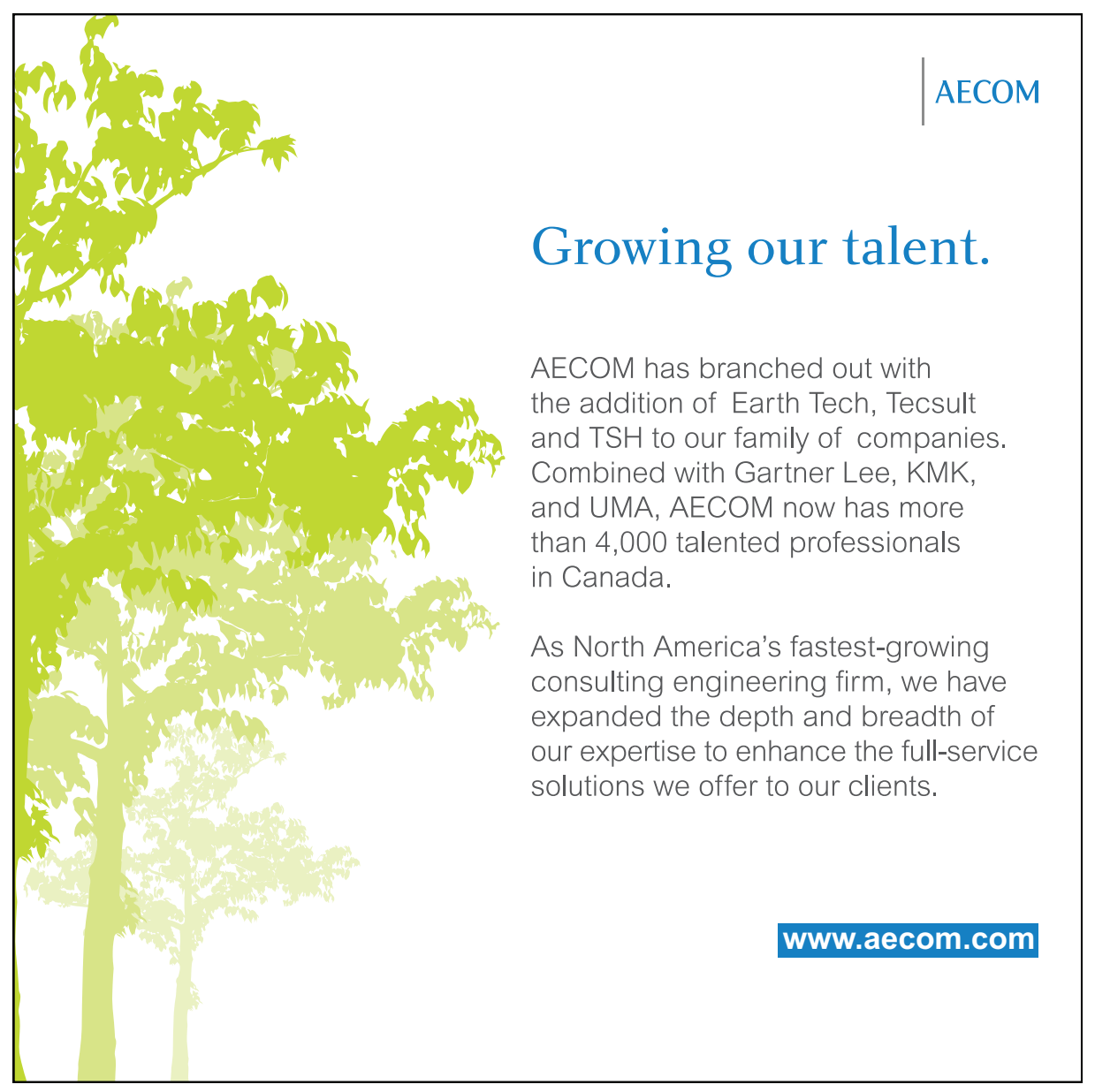

3 York University (2008). Space science fact sheet. Internet: research.yorku.ca/ _files/file.php? fileid = \&filename=file_ SpaceFactSheet.pdf (accessed August 5, 2008).

4 Landsburg, A.C. (2008). The art of successfully applying human systems integration (HSI). Internet: www.navalengineers. org/Events/HSIS2007/documents/ ANSEPaperdraft22ArtofHSI070411.pdf (accessed August 5, 2008).

5 Grech, M.R., Horberry, T.J., and Koester, T. (2008). Human factors in maritime domain. CRC Press, Boca Raton, Florida.

6 National Institute for Occupational Safety and Health (2008). Mining spotlight. Internet: www.cdc.gov/niosh/ mining (accessed August 5, 2008).

7 Sealy, J.W. (2008). Human factors What is that?! The American Institute of Architects, Internet: blog.aia. org/aiarchitect/2007/09/human_ factorswhat_is_that.html (accessed August 5, 2008).

8 Crews, J.E., and Campbell, V.A. (2001). Health conditions, activity limitations and participation restrictions among older people with visual impairments. J. Vision Impairment and Blindness, 95, 453-467.

9 Ball, K.K., Roenker, D., Wadley, V.G., Edwards, J.D., and Roth, D.L. (2006). Can high risk older drivers be identified through performance based measures in a department of motor vehicle setting? J. American Geriatrics Society, 54, 77-84.

${ }^{10}$ Marchau, V.A., and Walker, W.E. (2003). Dealing with uncertainty in implementing advanced driver assistance systems: An adaptive approach. Integrated Assessment, 4(1), 35-45.

${ }^{11}$ Easa, S.M., and He, W. (2006). Modeling driver visual demand on three-dimensional highway alignments. J. Transportation Engineering, 132(5), 357-365.

${ }^{12}$ Russo, F.A., Lantz, M.E., English, G.W., and Cuddy, L.L. (2003). Increasing effectiveness of train horns without increasing intensity. Proc., Inter. Conf. on Auditory Display, Boston, MA, 51-54.

${ }^{13}$ Russo, F.A., and Jones, J.A. (2007). Urgency is a non-monotonic function of pulse rate. J. Acoustical Society of America, 122(5), EL185-190. 